\title{
Micro-ramp Flow Control for Oblique Shock Interactions: Comparisons of Computational and Experimental Data
}

\author{
Stefanie M. Hirt ${ }^{1}$ \\ NASA Glenn Research Center, Cleveland, Ohio, 44135 \\ David B. Reich ${ }^{2}$ \\ University of Florida, Gainesville, Florida 32611 \\ and \\ Michael B. O'Connor ${ }^{3}$ \\ University of Notre Dame, Notre Dame, Indiana, 46556
}

\begin{abstract}
Computational fluid dynamics was used to study the effectiveness of micro-ramp vortex generators to control oblique shock boundary layer interactions. Simulations were based on experiments previously conducted in the $15 \times 15 \mathrm{~cm}$ supersonic wind tunnel at NASA Glenn Research Center. Four micro-ramp geometries were tested at Mach 2.0 varying the height, chord length, and spanwise spacing between micro-ramps. The overall flow field was examined. Additionally, key parameters such as boundary-layer displacement thickness, momentum thickness and incompressible shape factor were also examined. The computational results predicted the effects of the micro-ramps well, including the trends for the impact that the devices had on the shock boundary layer interaction. However, computing the shock boundary layer interaction itself proved to be problematic since the calculations predicted more pronounced adverse effects on the boundary layer due to the shock than were seen in the experiment.
\end{abstract}

\section{Nomenclature}

$A_{p} \quad=$ micro-ramp half angle

$c=$ micro-ramp chord length

$H=$ incompressible shape factor

$h \quad=$ micro-ramp height

$M \quad=$ Mach number

$s \quad=$ micro-ramp spacing

$u \quad=$ velocity component in the flow direction

$x=$ coordinate in the flow direction

$y^{+} \quad=$ distance from wall normalized by shear length scale

$z \quad=$ coordinate in the spanwise direction

$\alpha=$ shock generator angle of attack

$\delta \quad=$ compressible boundary-layer displacement thickness

$\theta=$ compressible boundary-layer momentum thickness

\section{Introduction}

TN supersonic inlets, oblique shocks are used to compress and decelerate the airflow entering the engine. These shocks interact with the boundary layer and increase its thickness, resulting in large energy losses, decreased system performance, and potentially separated flow. The traditional method to control shock boundary layer

${ }^{1}$ Aerospace Engineer, Inlet and Nozzle Branch, 21000 Brookpark Rd, M/S 5-12, AIAA Senior Member.

2 Student, Department of Mechanical and Aerospace Engineering, NASA USRP Intern.

${ }^{3}$ Student, Department of Aerospace and Mechanical Engineering, NASA USRP Intern.

1

American Institute of Aeronautics and Astronautics 
interactions (SBLIs) is bleed, which removes low-momentum flow from the boundary layer using suction through a porous surface on the inlet wall. Although bleed helps reduce the characteristic effects on the boundary layer, bleed systems are heavy and complex, decrease mass flow to the engine and introduce additional drag. Together these negative consequences of bleed lead to lower system efficiencies ${ }^{1,2}$.

Many different types of vortex generators (VGs) have been proposed and investigated as possible replacements or supplements to bleed systems ${ }^{3}$. VGs are devices that redistribute the energy through the boundary layer. The velocity near the wall is increased to help maintain boundary-layer health through the SBLI at the cost of a velocity defect in the outer region of the boundary layer. They are attractive due to their low weight and mechanical simplicity, though they do impose a drag penalty on the system.

Micro-ramps are one such type of VG flow control device. However, unlike typical vane-type vortex generators which have heights matching the boundary-layer thickness, micro-ramps have heights ranging from $25 \%$ to $40 \%$ of the boundary-layer thickness ${ }^{4}$. Micro-ramp style VGs have gained interest due to their mechanical robustness.

Experiments studying the effectiveness of micro-ramp flow control were performed in the $15 \mathrm{x} 15 \mathrm{~cm}$ supersonic wind tunnel at NASA Glenn Research Center (GRC) by Hirt and Anderson ${ }^{5}$. Fifteen micro-ramp configurations of varying height, chord length, and spanwise spacing between micro-ramps were investigated. A photograph showing three of the configurations from the experiment is shown in Figure 1. The 15 configurations represented a Design of Experiments (DOE) central composite design. An oblique shock was created using a shock generator plate at $8.5^{\circ}$ angle of attack. Micro-ramps were placed upstream of where the shock reflects off of the tunnel floor. Boundarylayer profiles and properties and Mach number contours were examined for various micro-ramp configurations.

This paper presents results of a computational fluid dynamics (CFD) study of micro-ramp effects on an oblique SBLI based on the experiments of Hirt and Anderson ${ }^{5}$. In this effort, the baseline cases and four cases comprising the main effects design-which is embedded in the complete central composite design from the experiment-were simulated in order to determine the ability of Reynolds-averaged Navier-Stokes (RANS)-based computation methods to predict the effects of micro-ramps. This paper discusses the CFD results and compares them with the experimental data.

\section{Modeling}

\section{A. Computational Methodology}

Simulations were conducted using Wind-US version $2.0^{6}$, a compressible 3-D RANS flow solver for multi-zone structured and unstructured grids. Wind-US includes several turbulence models, of which the Menter Shear Stress Transport (SST) model was chosen for this study. The SST turbulence model was chosen for its accuracy in wall boundary layers ${ }^{7}$. Structured grids were created using Pointwise Gridgen. A viscous grid was created using a wall grid spacing of 7.34E-4 $\mathrm{cm}$ based on an average $y^{+}$value of 2.0. A hyperbolic tangent distribution was used to distribute the grid points across the computational flow domain. A symmetry plane along the centerline of the tunnel was used to reduce the number of grid points by half, greatly reducing computational time. The grid was sequenced to reduce convergence time: the solution was first computed on a grid using every fourth point in each direction (coarse grid); then the solution was computed at every other point (medium grid); and finally the solution was computed using all the grid points (fine grid). Convergence was determined by examining differences in the parameters of interest-compressible displacement thickness, compressible momentum thickess, and incompressible shape factor-at the primary measurement plane, $x=4 \mathrm{~cm}$. If the values changed less than $0.5 \%$ over 2500 iterations, the solution was deemed converged. Boundary layer velocities, skin friction coefficient, and vorticity profiles were also monitored as a qualitative check on convergence.

\section{B. Configurations}

The seven configurations modeled for this effort are divided into two groups: three baseline cases and four complete cases with micro-ramps and an oblique shock. The three baseline cases were modeled to match the experimental baseline configurations. The first baseline configuration was the empty $15 \times 15 \mathrm{~cm}$ wind tunnel with a Mach 2.0 nozzle, which was simulated to verify all computational parameters were set up correctly (all subsequent simulations were also at $\mathrm{M}=2.0$ ). This grid contained 2.9 million grid points. For the second baseline configuration an oblique shock generator plate was added to the tunnel grid. The grid with the shock generator contained 5.9 million grid points. The final baseline configuration modeled the micro-ramps, but no shock generator, and contained 4.0 million grid points.

After the computations of the baseline cases were completed successfully, grids were built for the four microramp with shock cases. The micro-ramp/shock generator grids contained about 15 million grid points at the fine grid level. The four micro-ramp configurations tested are shown in Table 1 with the referenced parameters from the table 
shown in Figure 2. The micro-ramp half angle, $A_{p}$, was $24^{\circ}$ for all configurations. Micro-ramp centerlines $(c / 2)$ were placed at $x=-13 \mathrm{~cm}$, with the inviscid shock impingement location specified as $x=0 \mathrm{~cm}$ as shown in Figure 3 . The four micro-ramp configurations tested were the main effects configurations from the designed experiment of Hirt and Anderson ${ }^{5}$. The main effects design was chosen due to the ability to study many variables with a limited number of test cases since it represents the sparsest DOE design.

\section{Post Processing}

The data presented a challenge when computing boundary-layer parameters. There were density variations in the tunnel due to imperfections in the expansion from the nozzle, presenting a more complex boundary layer analysis. But in the cases with a shock, density had to be taken into account since the region downstream of the shock had no clear boundary layer edge when looking at $u$-velocity alone. For consistency, the boundary layer edge was defined as the location where either the streamwise velocity or streamwise momentum reached a maximum.

Since the intent of the micro-ramps is to improve the boundary layer, standard boundary layer properties were examined to determine their effectiveness. Specifically, the compressible displacement thickness, compressible momentum thickness, and incompressible shape factor are considered in this paper. The displacement and momentum thicknesses give a measure of the flow blockage added by the devices, while the shape factor measures the boundary layer health.

The incompressible shape factor, $H$, was chosen primarily because it has been used to quantify improvements in boundary layer health for bleed flows ${ }^{1}$. The incompressible shape factor is typically used instead of the compressible form even in compressible flow because it is independent of Mach number. A flat plate turbulent boundary layer has an incompressible shape factor of approximately 1.3 at all Mach numbers, and a boundary layer near separation has an incompressible shape factor of about $2.7^{8}$.

\section{Results}

\section{A. Baseline Cases}

\section{Empty Tunnel}

The $u$-velocity contours of the empty tunnel baseline simulation are shown in Figure 4 . The maximum velocity in the core flow is $519 \mathrm{~m} / \mathrm{s}$, which given the speed of sound of $258 \mathrm{~m} / \mathrm{s}$ gives the expected Mach number of 2.0. Figure 5 shows a comparison of boundary-layer u-velocity profiles for the three grid sequences and the experimental data. All of the cases predict the correct velocity in the freestream. With grid refinement, the CFD solutions closely match the experiment throughout the boundary layer. Only subtle changes are observed between the medium and fine grid solutions, indicating grid convergence was obtained. Table 2 and Figure 6 (a) and (b) show the baseline boundary-layer integral parameters compared with experiment. As expected, for the case with a simple convergingdiverging nozzle and flat plate boundary layer, the agreement of the computed boundary layer parameters and growth rate to the measured values was very good.

\section{Shock Only: Viscous Sidewalls}

Attempts to run simulations for the $8.5^{\circ}$ shock generator angle were unsuccessful in maintaining a supersonic flow within the vicinity of the shock generator. The pressure rise from the shock generator would move upstream of the shock generator, and the tunnel flow would unstart as in a supersonic inlet. This was caused by boundary layer thickening along the tunnel floor coupled with an even stronger adverse flow response along the sidewall. Corrective measures such as starting the calculations with a higher inflow pressure (to increase the Reynolds number and reduce the effective blockage) always reverted to the same unstarted flow state when the pressures were reduced to the experimental conditions. In addition to the SST turbulence model this case was run using the Spalart-Allmaras (SA) turbulence model, which tends to predict weaker SBLIs. However, no significant improvement was noted when the SA turbulence model was used. In subsequent attempts, the shock generator angle was first reduced to $7.5^{\circ}$, which still exhibited the undesirable unstart behavior, and then ultimately reduced to $6.5^{\circ}$, which eliminated the unstart characteristics, yet continued to produce a pressure rise upstream of the leading edge of the shock generator plate.

Even for the $6.5^{\circ}$ shock generator case, when the micro-ramps were introduced, the effect of the sidewall boundary layer thickening interacted with the wake of the micro-ramps nearest the sidewall causing the flow to unstart. As a result, a further deviation from the exact experimental configuration was made to remove the viscous effect of the sidewall boundary layers to isolate the effects of the micro-ramps on the SBLI. This was accomplished by changing the sidewall boundary condition from viscous wall to inviscid wall. While no longer accurately representing the experimental configuration, comparisons between CFD and experiment were restricted to a small 
region (17\% to $23 \%$ of the test section width depending on the micro-ramp spacing) near the center of the tunnel where, in the experiment, the flow was observed to be free of tunnel sidewall effects.

The computational grid and contours of u-velocity are shown in Figure 7 for the tunnel with shock generator installed at $6.5^{\circ}$ angle of attack. Figure 8 illustrates the interaction between the sidewall boundary layer and the tunnel floor boundary layer due to the shock. Measurements were not taken near the sidewalls in the experiment, but this sidewall boundary layer thickening was expected and has been observed in similar experiments.

\section{Shock Only: Inviscid Sidewalls}

In order to mitigate the viscous wall issue and better observe the micro-ramp effectiveness, the tunnel sidewall boundary condition was changed from viscous wall to inviscid wall. All cases were simulated with this modified boundary condition, including the baseline cases. The tunnel floor and ceiling remained viscous to preserve the SBLI of interest. Figure 9 shows the shock-only baseline case with inviscid sidewall. It is apparent that the excessive sidewall boundary layer thickening is eliminated and no shocks are present upstream of the shock generator plate.

From Figure 9 it is apparent that the boundary layer is significantly thickened in the region where the oblique shock impacts the tunnel wall. This illustrates the SBLI that is to be mitigated by the micro-ramps.

\section{Micro-ramps Only: Inviscid Sidewalls}

This simulation showed the vortices generated by micro-ramp configuration 2 unaffected by any shocks. The grid in the vicinity of the micro-ramps is shown in Figure 10. Figure 11 illustrates the vortices formed by flow over the micro-ramps. As shown in Figure 11, vorticity magnitude is highest near the trailing-edge tip of the micro-ramps and decays downstream. Previous work has shown that vorticity decay occurs at a higher rate in RANS-based CFD than is measured experimentally ${ }^{9,10}$. As shown in Table 2, the micro-ramps tend to increase compressible displacement thickness compared to the empty tunnel cases at the corresponding locations, but have little effect on incompressible shape factor when no shock is present. Figure 6 (c) and (d) show the micro-ramp only baseline CFD compared to the experimental data. Agreement is generally good expect for the first station downstream of the microramps at $\mathrm{x}=-8 \mathrm{~cm}$. Because this station is so near the micro-ramps, it may be difficult to obtain good measurements in the experiment and calculate good velocities in the computational results.

\section{B. Micro-ramps With Shock}

The inviscid wall boundary condition was applied to the tunnel sidewalls for all CFD simulations of the tunnel with the shock generator and micro-ramps. The viscous wall boundary condition continued to be applied to the upper and lower walls of the tunnel, as the SBLI on these surfaces was of primary interest in this study. Figures 12 and 13 show $u$-velocity contours of the four micro-ramp/shock configurations. Local separation occurs in the wake region of each micro-ramp, however it is unclear from contour plots alone whether flow is improved in the spanwise region between the micro-ramps. Spanwise averaging of boundary-layer parameters was used to obtain a qualitative measure of micro-ramp effectiveness. Table 3 shows compressible displacement thickness, compressible momentum thickness, and incompressible shape factor for all four micro-ramp with shock configurations as well as the shock only baseline case for comparison. Compared to the shock-only case, displacement thickness and momentum thickness increased with the addition of micro-ramps. The incompressible shape factor decreased for three of the four micro-ramp configurations.

Data were available from the $15 \times 15 \mathrm{~cm} \mathrm{SWT}$ test for one micro-ramp configuration with a shock generator angle of $6.5^{\circ}$. and was modeled as configuration 4 in the current study. The CFD and experimental data are presented in Table 4. Also presented are the experimental results for that configuration at $7.5^{\circ}$ and $8.5^{\circ}$ shock generator angle. Again the CFD predicts an effect of the shock interaction stronger than that observed in the experiment in terms of increased boundary-layer parameter thicknesses and increased shape factor. In fact, the computed boundary-layer parameters are closest to the experimental data at $8.5^{\circ}$. The experimental data seems to show a distinct break where the data at $6.5^{\circ}$ and $7.5^{\circ}$ are quite similar with a slight increase in the displacement and momentum thicknesses, $\delta^{*}$ and $\theta$, without a change in shape factor, $H$, but the values of all three parameters for the $8.5^{\circ}$ case are significantly increased. It is possible that this is indicative of the flow being attached for the two small shock generator angles and separated at the highest angle. If this is the case, then it seems that the CFD is predicting an earlier separation limit in terms of shock strength than is evidenced in the experimental data.

To compare the trends between micro-ramp configurations, Figure 14 shows the span-averaged displacement and momentum thicknesses, $\delta^{*}$ and $\theta$, and shape factor, $H$, for the CFD compared to experimental data. Experimental data is shown in blue for all four micro-ramp configurations at a shock generator angle of $8.5^{\circ}$ and in green for configuration 4 with a shock generator angle of $6.5^{\circ}$. The computational results are shown in red with squares marking the four configurations and the horizontal line representing the value for the $6.5^{\circ}$ shock-only 
baseline. Despite the simulations not exactly modeling the experimental configuration, the flow affected by the micro-ramps away from the sidewalls was successfully simulated and captured the trends of the impact of the ramps on the shock system, especially in terms of the shape factor. It is important to mention, however, that the CFD predictions represent a $6.5^{\circ}$ shock generator angle, while the experimental data was obtained with a shock generator angle of $8.5^{\circ}$. We would expect that the CFD prediction for the $6.5^{\circ}$ case should match the experimental data for the same angle. However, we observe that the values more closely match those for the $8.5^{\circ}$ case, suggesting that the CFD predicts a greater adverse response to the SBLI than seen in the experiment.

\section{Conclusions}

The primary challenge encountered in the present study was that the CFD solutions showed limitations in replicating experimental results with the oblique shock, especially when the sidewalls were modeled as viscous walls. Across the board, the CFD predicted a greater adverse response to the shock interaction than was observed in the experiment. This was seen as a tunnel unstart with a plate angle of $8.5^{\circ}$ and also as a larger boundary-layer shape factor with a plate angle of $6.5^{\circ}$. Even when the sidewalls were modeled as inviscid walls, the response of the boundary layer to the shock interaction is not captured well by currently available RANS-based CFD.

Although there were difficulties modeling the exact experimental configuration, the modified simulations with inviscid sidewalls are a useful complement to the experimental data. The effect of the micro-ramps on the shock showed the same trends as for the experiment based on the limited data available for the $6.5^{\circ}$ case. The addition of micro-ramps to a SBLI reduced the incompressible shape factor in three of the four configurations. However it is still unclear whether the micro-ramps are beneficial since both the displacement thickness and momentum thickness increased with the addition of micro-ramps. This trade-off would need to be considered relative to a specific inlet system.

For the cases without a shock present, the CFD agreed reasonably well with the experiment. This was even true for the case with only the micro-ramps even though there are some questions as to whether a RANS solver is appropriate to use with vortical flows. Indeed, the RANS approximation needs to be more closely examined in its application to the study of vortical flow, micro-ramps in particular. All RANS models respond to vorticity by increasing turbulent viscosity, in turn decreasing vorticity, possibly too fast (vorticity is not preserved far enough downstream) to represent a real physical flow. Other approaches such as large-eddy simulation or direct numerical simulation should be investigated and may give more informative results.

\section{Acknowledgments}

The authors would like to thank Dr. Nicholas Georgiadis for his time and advice. This work was supported by the Supersonics project under NASA's Fundamental Aeronautics Program.

\section{References}

${ }^{1}$ Fukuda, M. K., Hingst, W. G., Reshotko, E., "Control of Shock Wave-Boundary Layer Interactions by Bleed in Supersonic Mixed Compression Inlets," NASA CR2595, 1975.

${ }^{2}$ Young, D. D., Jenkins, S. A., "An Investigation of Active Flowfield Control for Inlet Shock/Boundary Layer Interaction," AIAA Paper 2005-4020, July 2005.

${ }^{3}$ Lin, J. C., "Review of Research on Low-Profile Vortex Generators to Control Boundary-Layer Separation," Progress in Aerospace Sciences, Vol. 38, 2002, pp. 389-420.

${ }^{4}$ Anderson, B.H., Tinapple, J., Surber, L., "Optimal Control of Shock Wave Turbulent Boundary Layer Interactions Using Micro-Array Actuation,” AIAA Paper 2006-3197, June 2006.

${ }^{5}$ Hirt, S.M., Anderson, B.H., "Experimental Investigation of the Application of Microramp Flow Control to an Oblique Shock Interaction," AIAA Paper 2009-919, Jan 2009.

${ }^{6}$ Towne, C. E., "Wind-US Users Guide, Version 2.0," NASA/TM-2009-215804, 2009.

${ }^{7}$ Georgiadis, N.J., Yoder, D.A., Towne, C.S., Engblom, W.A., Bhagwandin, V.A., Power, G.D., Lankford, D.W., Nelson, C.C., "Wind-US Code Physical Modeling Improvements to Complement Hypersonic Testing and Evaluation," AIAA Paper 2009-193, Jan 2009.

${ }^{8}$ Castillo, L., Wang, X., George, W. K., "Separation Criterion for Turbulent Boundary Layers Via Similarity Analysis", Journal of Fluids Engineering, Vol. 126, 2004, pp 297-304.

${ }^{9}$ Dippold, V., "CFD Analyses and Jet-Noise Predictions of Chevron Nozzles With Vortex Stabilization,” AIAA Paper 200837, Jan 2008.

${ }^{10}$ Dudek, J. C., "Modeling Vortex Generators in the Wind-US Code," AIAA Paper 2010-32, Jan 2010. 
Tables

Table 1. Test matrix for micro-ramp configurations.

\begin{tabular}{|c|c|c|c|}
\hline Configuration & $h, \mathrm{~mm}$ & $c, \mathrm{~mm}$ & $s, \mathrm{~mm}$ \\
\hline 1 & 3 & 24 & 35 \\
\hline 2 & 3 & 12 & 25 \\
\hline 3 & 5 & 12 & 35 \\
\hline 4 & 5 & 24 & 25 \\
\hline
\end{tabular}

Table 2. Span-averaged compressible displacement thickness, compressible momentum thickness and incompressible shape factor for the baseline cases.

\begin{tabular}{|c|c|c|c|c|c|c|c|}
\hline & & \multicolumn{3}{|c|}{ Computational Results } & \multicolumn{3}{|c|}{ Experimental Results } \\
\hline & & $\delta^{*}, \mathrm{~cm}$ & $\theta, \mathrm{cm}$ & $H$ & $\delta^{*}, \mathrm{~cm}$ & $\theta, \mathrm{cm}$ & $H$ \\
\hline$x=-13 \mathrm{~cm}$ & empty tunnel & 0.301 & 0.101 & 1.30 & 0.283 & 0.100 & 1.32 \\
\hline \multirow{2}{*}{$x=-8 \mathrm{~cm}$} & empty tunnel & 0.321 & 0.104 & 1.30 & 0.292 & 0.104 & 1.31 \\
\hline & micro-ramps ${ }^{*}$ & 0.337 & 0.110 & 1.30 & 0.291 & 0.117 & 1.34 \\
\hline \multirow{2}{*}{$x=-4 \mathrm{~cm}$} & empty tunnel & 0.324 & 0.112 & 1.31 & 0.301 & 0.106 & 1.32 \\
\hline & micro-ramps & 0.347 & 0.111 & 1.29 & 0.324 & 0.108 & 1.30 \\
\hline \multirow[t]{2}{*}{$x=0 \mathrm{~cm}$} & empty tunnel & 0.333 & 0.115 & 1.30 & 0.342 & 0.116 & 1.30 \\
\hline & micro-ramps & 0.348 & 0.118 & 1.30 & 0.347 & 0.120 & 1.30 \\
\hline \multirow[t]{3}{*}{$x=4 \mathrm{~cm}$} & empty tunnel & 0.347 & 0.115 & 1.30 & 0.326 & 0.118 & 1.32 \\
\hline & micro-ramps & 0.356 & 0.118 & 1.29 & 0.337 & 0.124 & 1.32 \\
\hline & shock $^{* *}$ & 0.495 & 0.170 & 1.81 & 0.516 & 0.190 & 1.75 \\
\hline
\end{tabular}

Table 3. Span-averaged compressible displacement thickness, compressible momentum thickness and incompressible shape factor for micro-ramps with shock cases. $x=4 \mathrm{~cm}$.

\begin{tabular}{|c|c|c|c|}
\hline Configuration & $\delta^{*}, \mathrm{~cm}$ & $\theta, \mathrm{cm}$ & $H$ \\
\hline shock & 0.495 & 0.170 & 1.81 \\
\hline 1 & 0.520 & 0.183 & 1.75 \\
\hline 2 & 0.541 & 0.184 & 1.81 \\
\hline 3 & 0.546 & 0.192 & 1.77 \\
\hline 4 & 0.514 & 0.194 & 1.66 \\
\hline
\end{tabular}


Table 4. Span-averaged compressible displacement thickness, compressible momentum thickness and incompressible shape factor for micro-ramps with shock configuration 4 (CFD) compared to experimental data (SWT) for the same micro-ramp configuration at various shock generator angles. $x=4 \mathrm{~cm}$

\begin{tabular}{|c|c|c|c|c|}
\hline$\alpha$, degrees & & $\delta^{*}, \mathrm{~cm}$ & $\theta, \mathrm{cm}$ & $H$ \\
\hline 6.5 & CFD & 0.514 & 0.194 & 1.66 \\
\hline 6.5 & SWT & 0.400 & 0.129 & 1.29 \\
\hline 7.5 & SWT & 0.445 & 0.140 & 1.29 \\
\hline 8.5 & SWT & 0.556 & 0.226 & 1.62 \\
\hline
\end{tabular}

\section{Figures}

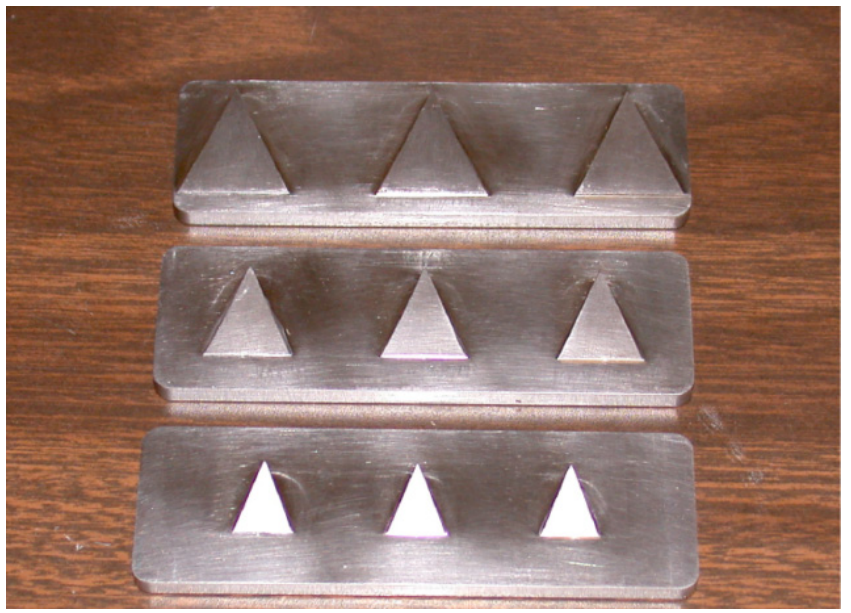

Figure 1. Picture of three micro-ramp inserts from the experiment in the 15 x $15 \mathrm{~cm}$ supersonic wind tunnel. 

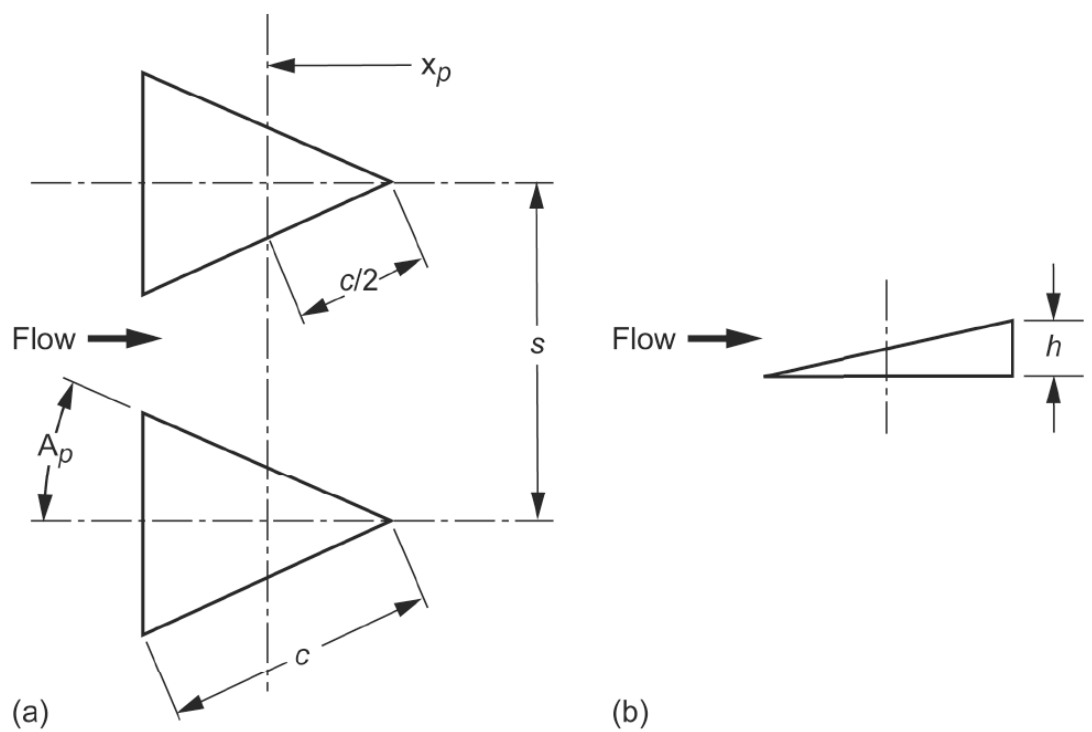

(b)

Figure 2. Micro-ramp configuration schematic.

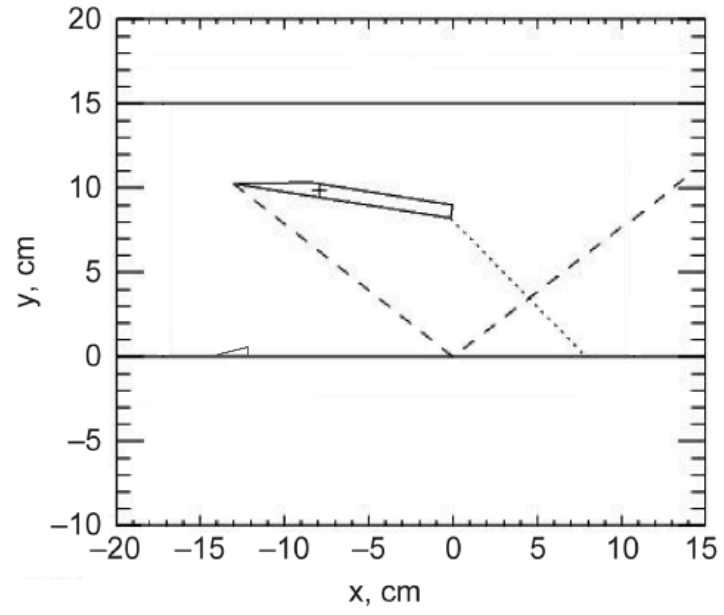

Figure 3. Tunnel layout. Dotted lines represent shocks and expansions.

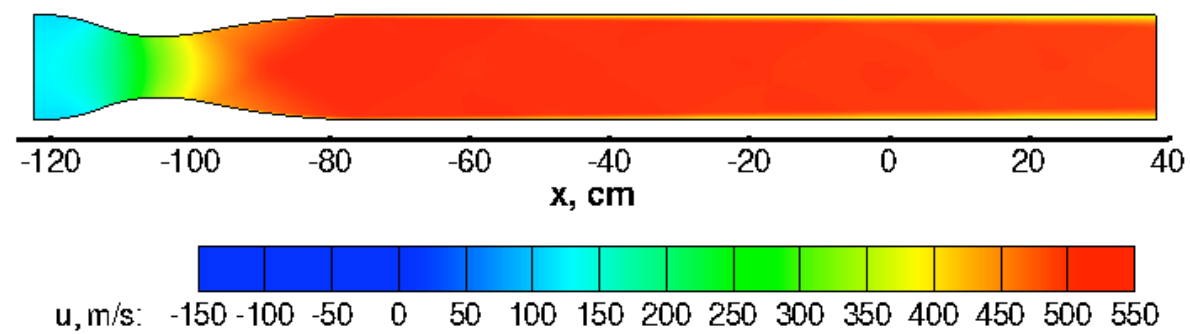

Figure 4. Contours of $u$-velocity for the empty $15 \times 15 \mathrm{~cm}$ supersonic wind tunnel configuration at $z=0 \mathrm{~cm}$. 


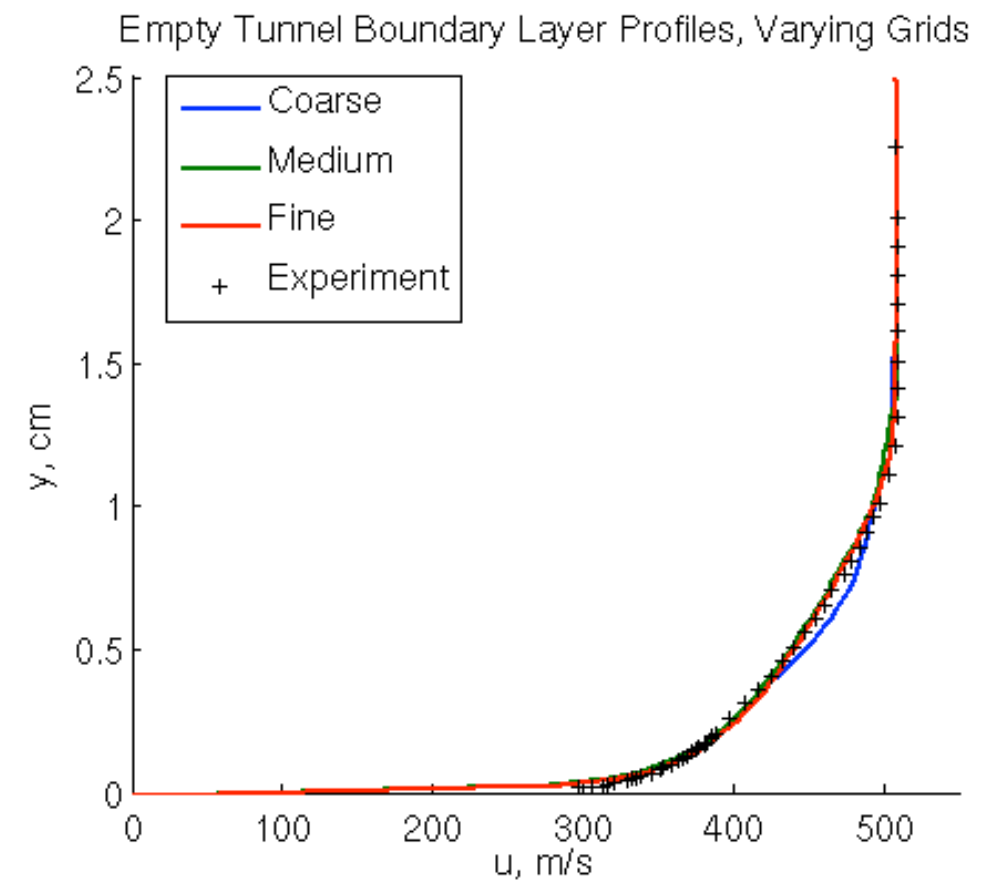

Figure 5. Grid sensitivity and experimental data for empty tunnel.

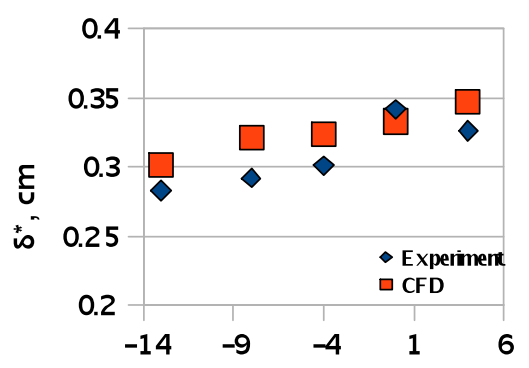

(a)

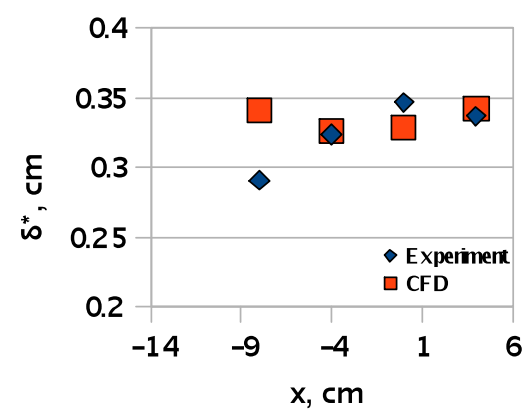

(c)

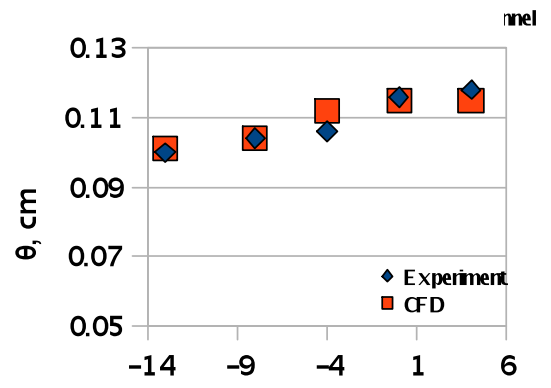

(b)

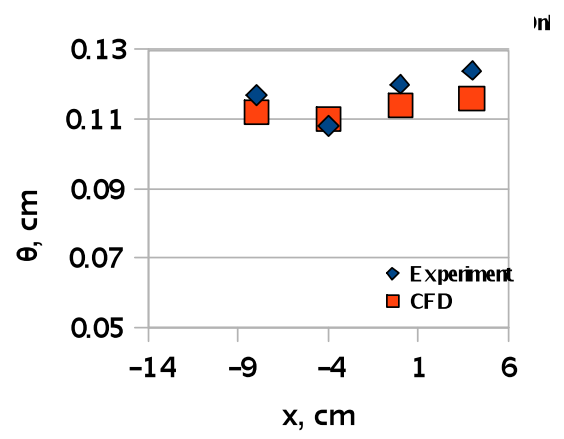

(d)

Figure 6. (a) Empty tunnel compressible displacement thickness, (b) empty tunnel compressible momentum thickness, (c) micro-ramps only compressible displacement thickness and (d) micro-ramps only compressible momentum thickness along the streamwise direction. 


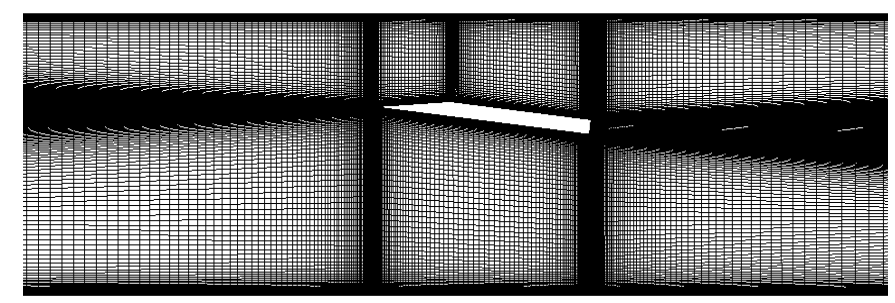

(a)

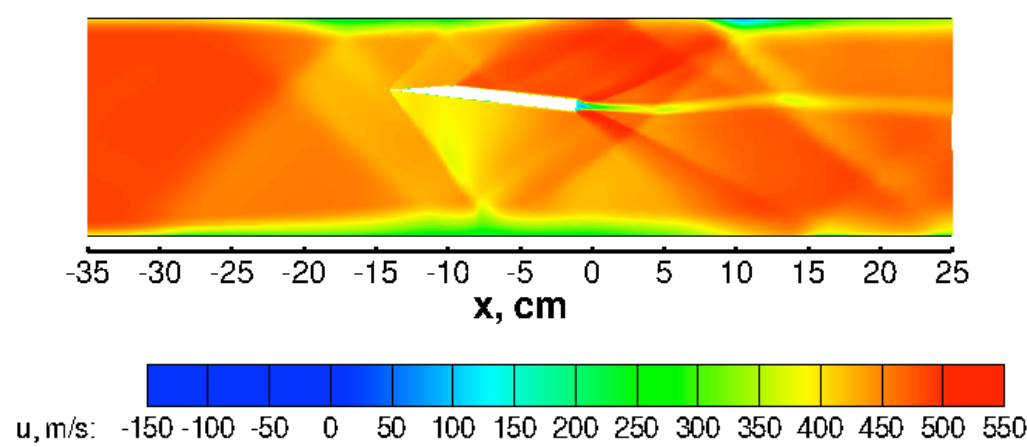

(b)

Figure 7. (a) Grid and (b) $u$-velocity contours for tunnel with shock generator plate at $6.5^{\circ}$ with viscous sidewalls at $z=0 \mathrm{~cm}$.

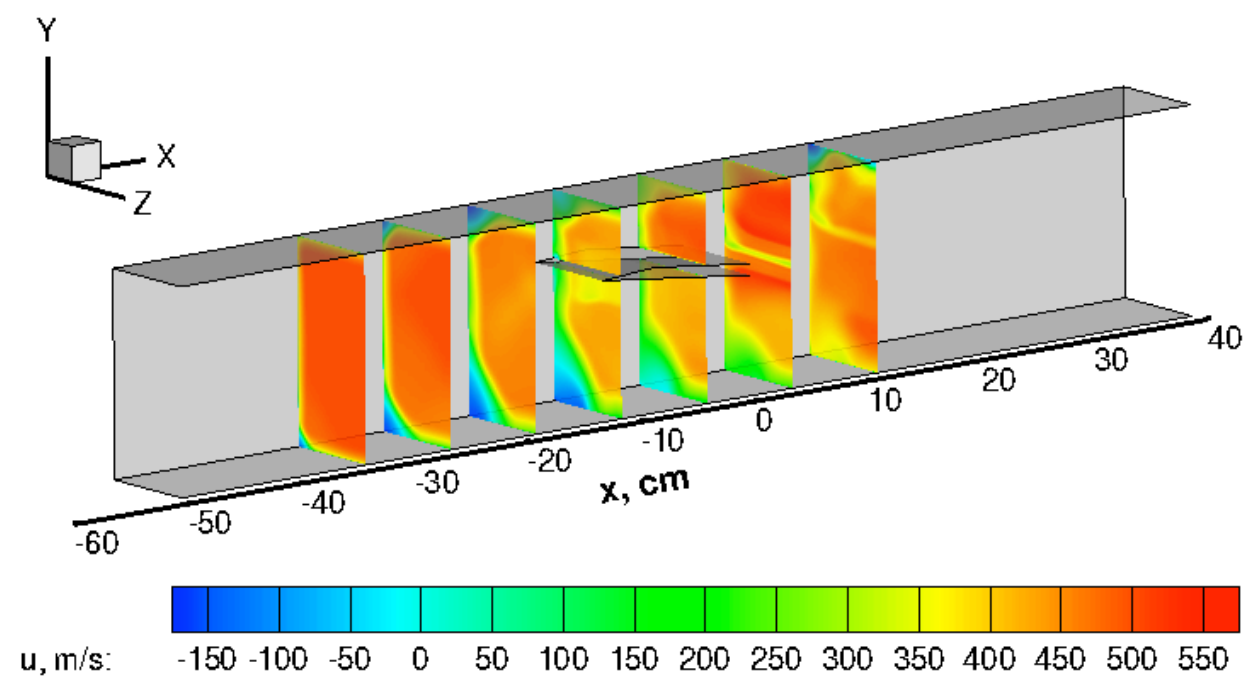

Figure 8. Contours of $u$-velocity at various streamwise locations for shock-only case with viscous sidewalls. Symmetry plane normal to the $z$-direction. 


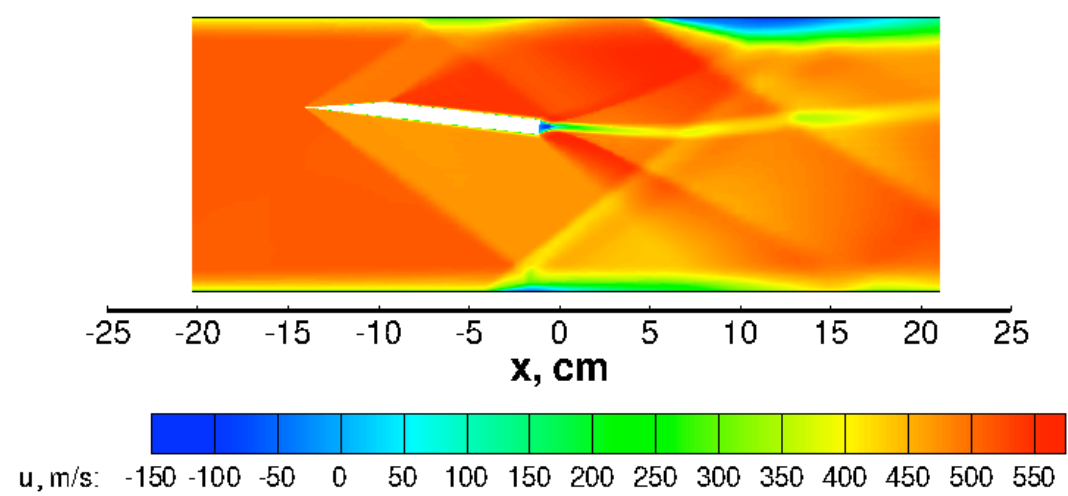

Figure 9. Contours of $u$-velocity for the shock-only case with inviscid tunnel sidewalls at $z=0 \mathrm{~cm}$.

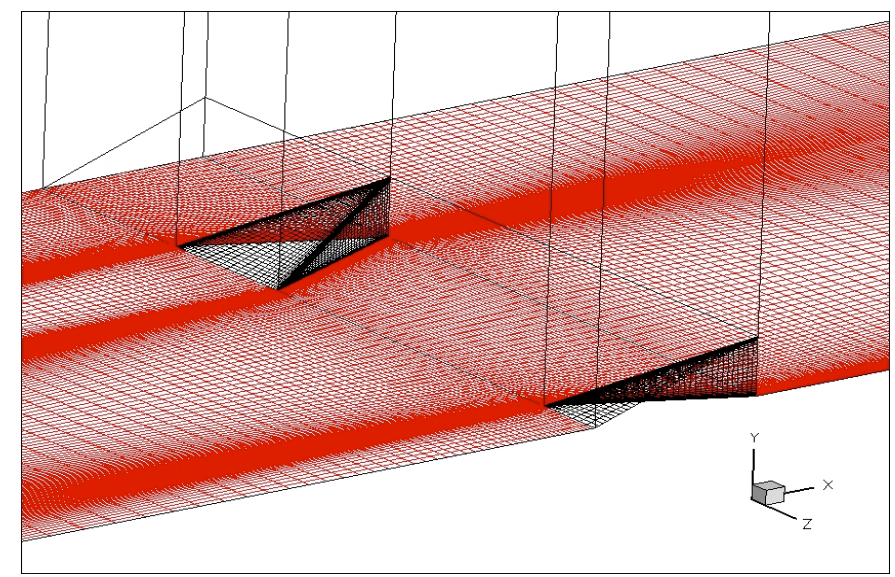

Figure 10. Grid in the vicinity of the micro-ramps. Flow is from left to right. Symmetry plane normal to $z$ direction.

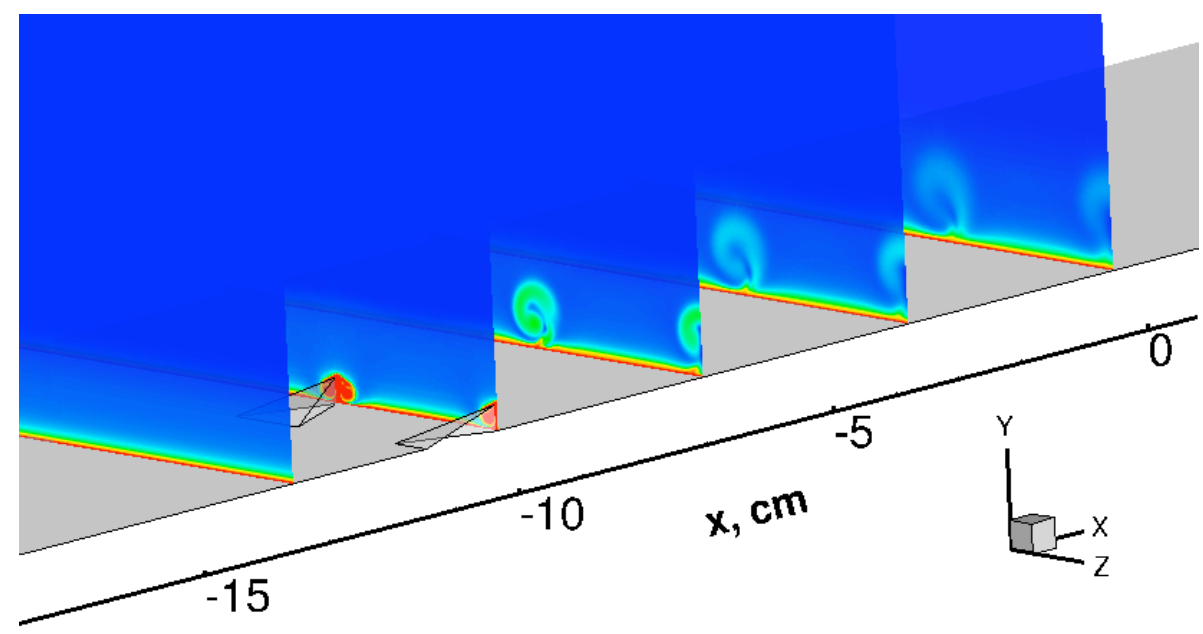

Nondimensional Vorticity Magnitude

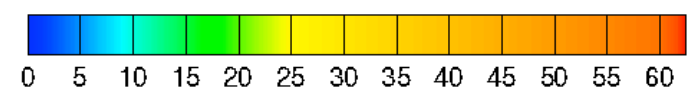

Figure 11. Vorticity magnitude contours at various streamwise locations. Flow is from left to right. Symmetry plane normal to $z$-direction. 


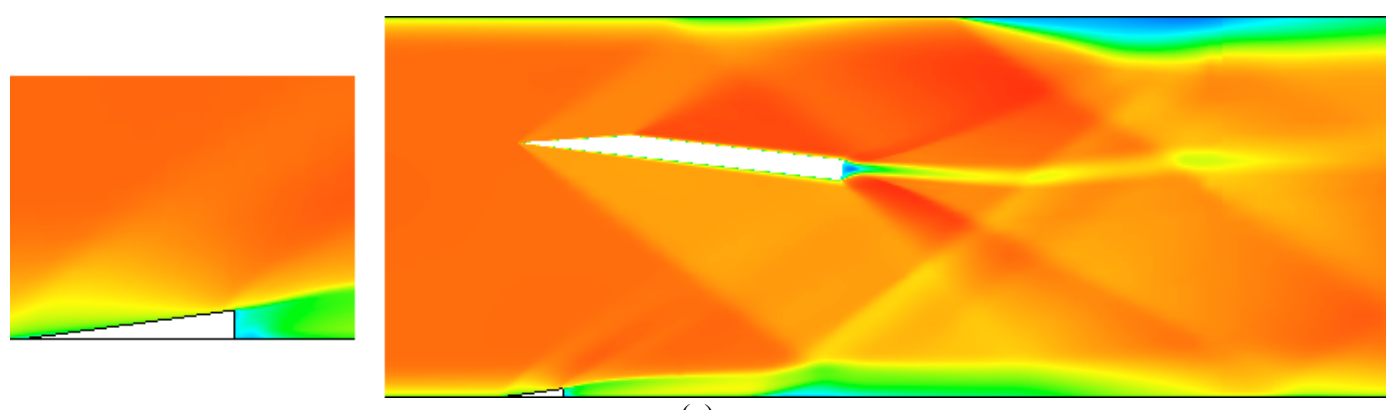

(a)

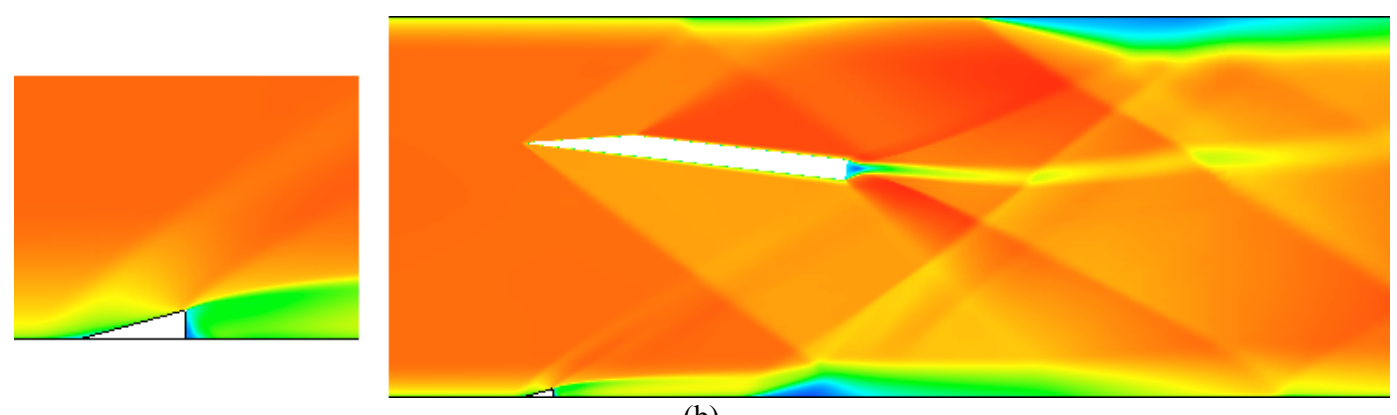

(b)

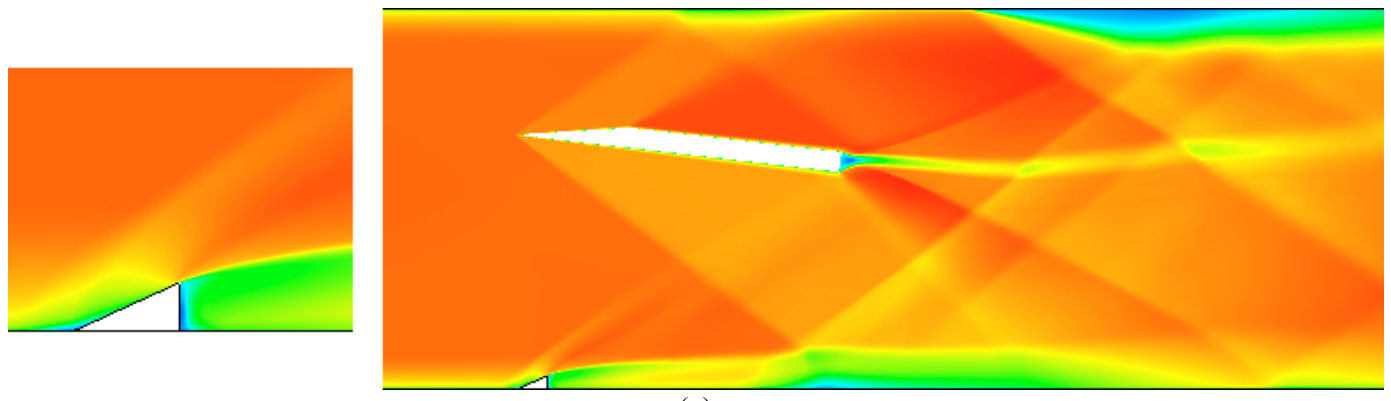

(c)

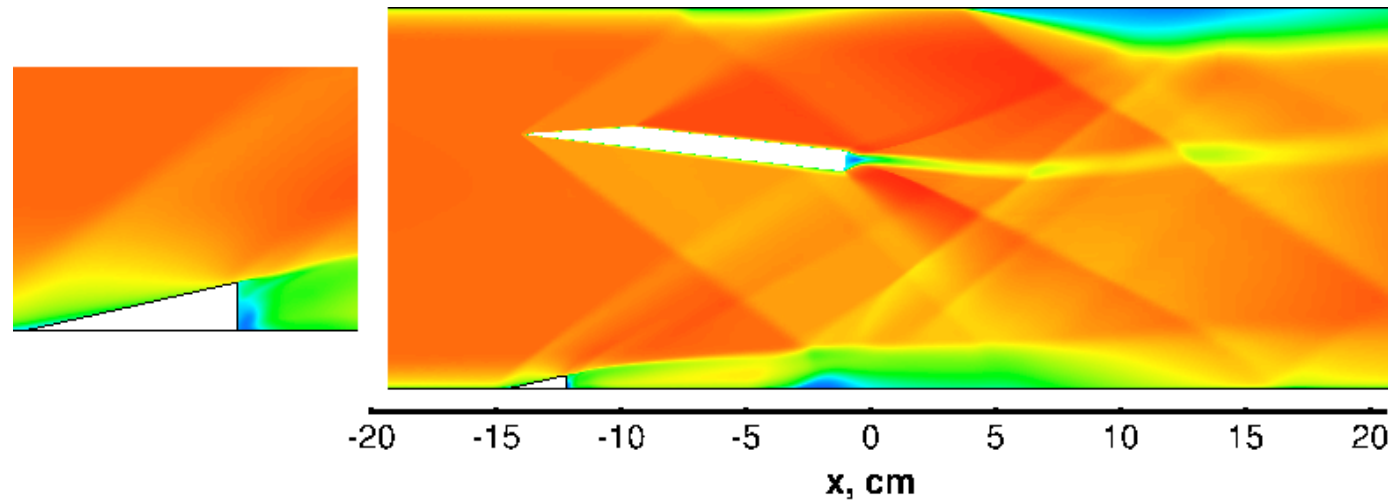

(d)

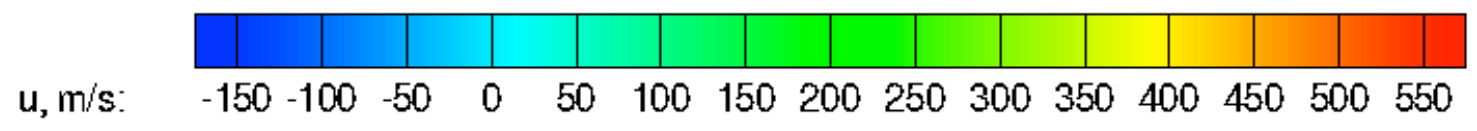

Figure 12. Contours of $u$-velocity at the tunnel centerline $(z=0)$ for the four micro-ramp configurations: a) Configuration 1, b) Configuration 2, c) Configuration 3, and d) Configuration 4. 
(a)

(b)

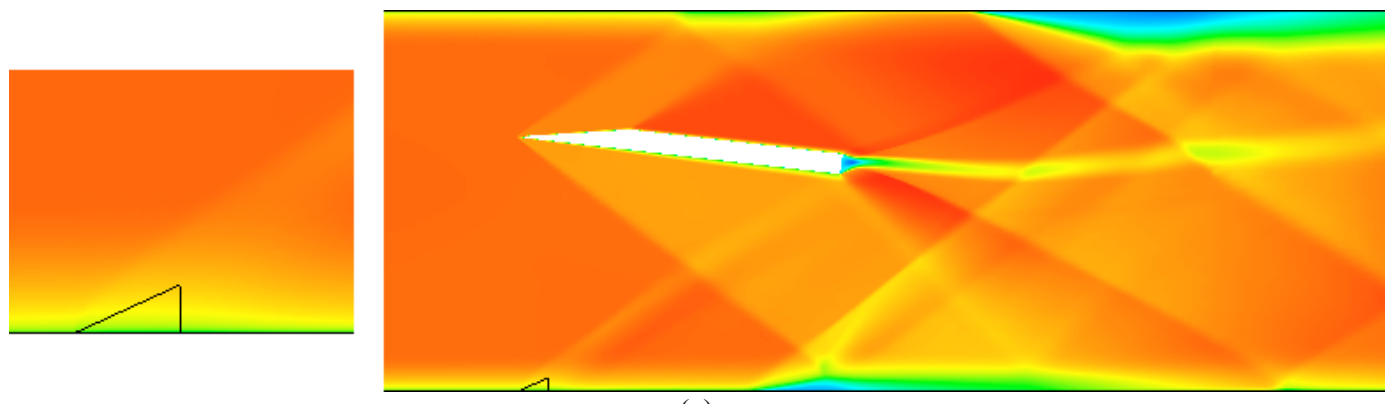

(c)

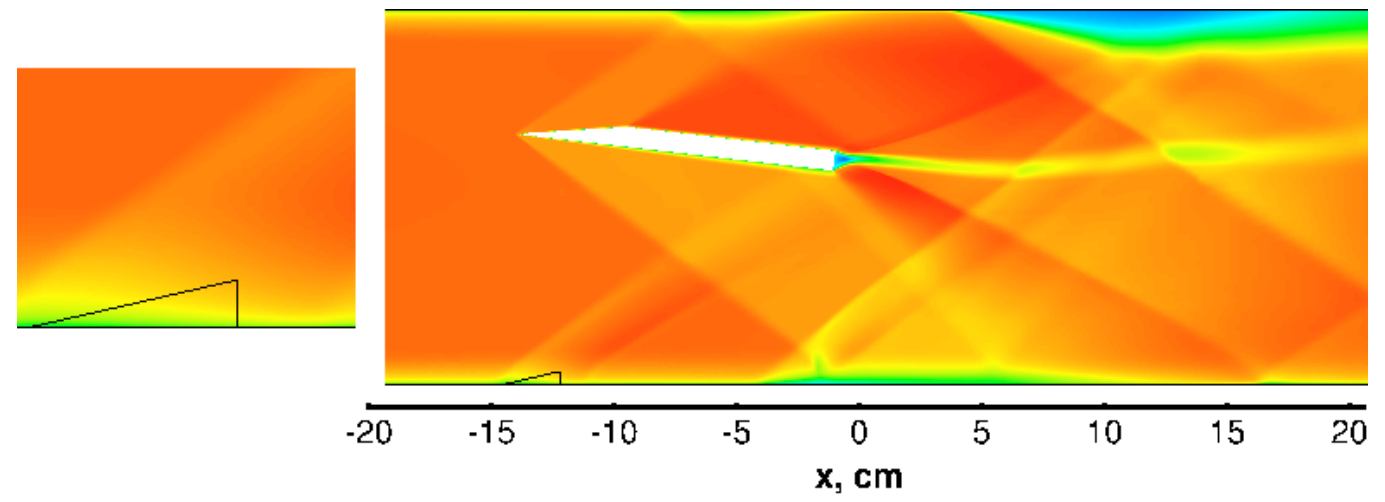

(d)

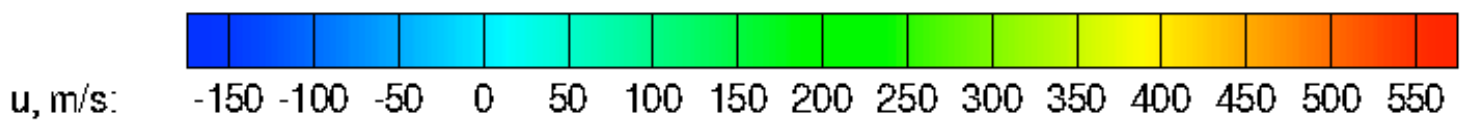

Figure 13. Contours of $u$-velocity halfway between the micro-ramps $(z=s / 2)$ for the four microramp configurations: a) Configuration 1, b) Configuration 2, c) Configuration 3, and d) Configuration 4. 

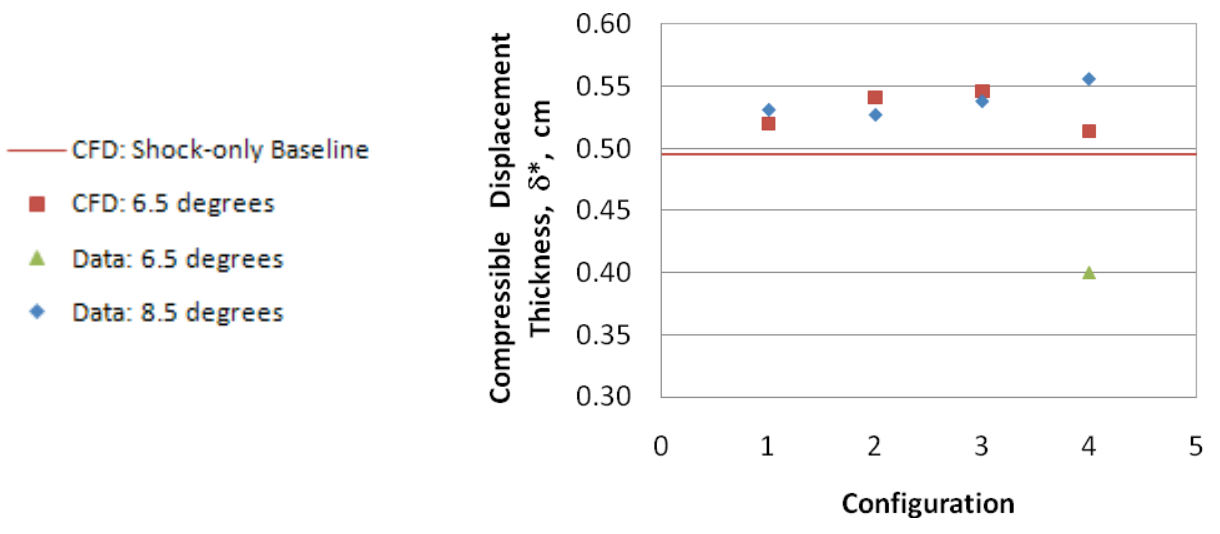

(a)

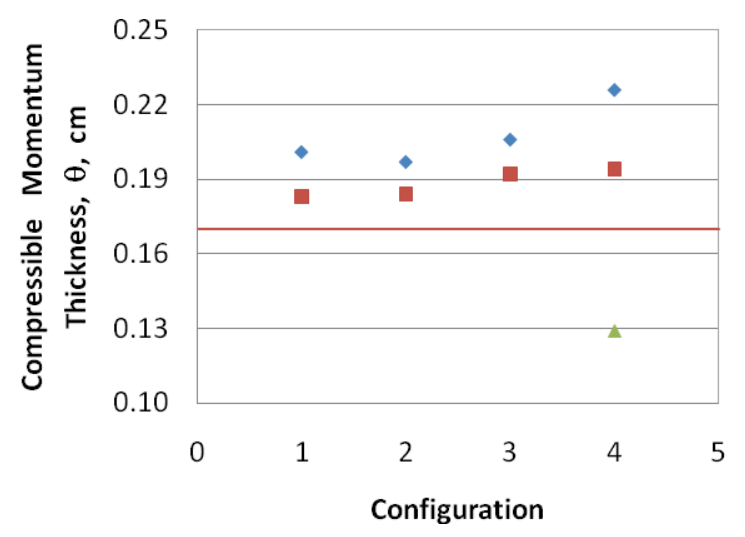

(b)

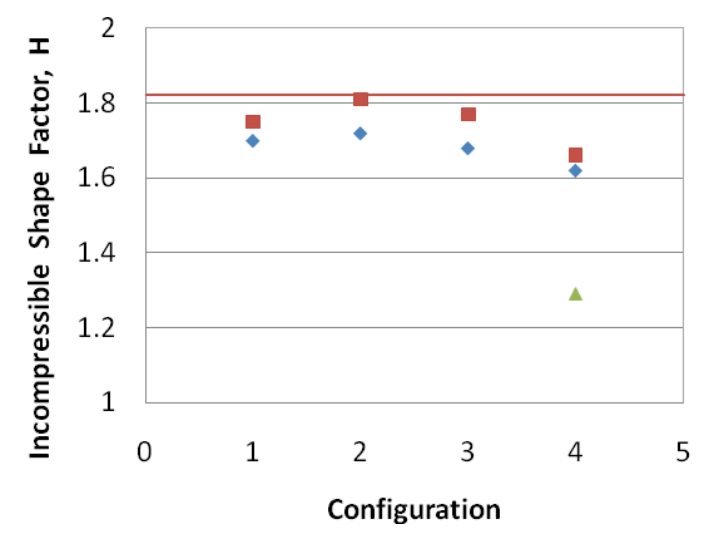

(c)

Figure 14. Plots showing comparisons of span-averaged boudary-layer parameters at $x=4 \mathrm{~cm}$ by configuration numbers from Table 1 along the $x$-axis: a) compressible displacement thickness, $\delta^{*}$, b) compressible momentum thickness, $\theta$, and c) incompressible shape factor, $H$. 